Z. Klin. Chem. Klin. Biochem.

12. Jg. 1974, S. 59-61

\title{
Bestimmung der Galaktose-1-phosphat-Uridylyltransferase-Aktivität im Erythrocytenhämolysat von Patienten mit Down-Syndrom ${ }^{1)}$
}

\author{
Von J. Nothjunge, Ingrid Most, Ingrid Hass und F. Menne
}

\author{
Aus der Abteilung für Stoffwechselforschung (Vorstand: Prof. Dr. F. Menne) des Physiologisch-Chemischen \\ Institutes der Universität Münster
}

(Eingegangen am 7. September/6. Dezember 1973)

Bei 28 Patienten mit Down-Syndrom (19 männlichen und 9 weiblichen Geschlechtes) wurde die Aktivität der Galaktose-1-phosphatUridylyltransferase (E.C. 2.7.7.12) im Erythrocytenhämolysat gemessen. Sie betrug $500 \mathrm{bis} 883 \mathrm{mU} / \mathrm{g} \mathrm{Hb}$ mit einem arithmetischen Mittelwert von $\bar{x}=690$ bei einer Standardabweichung von $s= \pm 103,50 \mathrm{mU} / \mathrm{g} \mathrm{Hb}$. Alters- und geschlechtsentsprechende Gesunde hatten im Erythrocytenhämolysat eine Enzymaktivität von 133 bis $483 \mathrm{mU} / \mathrm{g} \mathrm{Hb}(\overline{\mathbf{x}}=291, \mathrm{~s}= \pm 103,33 \mathrm{mU} / \mathrm{g} \mathrm{Hb})$. Damit lag die Aktivität dieses Enzymes bei Mongoloiden durchschnittlich um das 2,7-fache (Bereich: 1,0 bis 4,5, $s= \pm 1,05$ ) höher als bei Gesunden.

\section{Determination of the Galactose-1-phosphate-Uridylyltransferase activity in the erythocyte haemolysate of mongoloids}

The activity of Galactose-1-phosphate-Uridylyltransferase was measured in the erythrocyte haemolysate of 28 patients with Down's syndrome (19 males, 9 females). It was found to be between 500 and $883 \mathrm{mU} / \mathrm{g} \mathrm{Hb}$ with the mathematical mean of $\bar{x}=690$, and a standard deviation of $\pm 103.50 \mathrm{mU} / \mathrm{g} \mathrm{Hb}$. A control group of an equivalent number of healthy males and females with the same age range showed an enzyme activity of 133 to $483 \mathrm{mU} / \mathrm{g} \mathrm{Hb}(\bar{x}=291, \mathrm{~s}= \pm 103.33 \mathrm{mU} / \mathrm{g} \mathrm{Hb})$. Thus, the enzyme activity of the mongoloids was on average 2.7 times (range: 1.0 to $4.5, \mathrm{~s}= \pm 1.05$ higher than the control group.

Uber Enzymaktivitätssteigerung in den Leukocyten und Erythrocyten von Patienten mit Morbus Down liegen mehrere Berichte vor. Eine Übersicht geben $H$ sia et al (1) und Lenz (2). Bezüglich der Galaktose-1-phosphatUridylyltransferase (E. C. 2.7.7.12) sind diese Befunde jedoch nicht einheitlich. Brandt et al $(3,4)$, Hsia et al (5) und Rosner et al (6) fanden überhöhte Galaktose-1phosphat-Uridylyltransferaseaktivitäten im Vollblut, sowie Hsia et al (5), Mellman et al (7) und Rosner et al (6) in den Leukocyten. Während $\mathrm{Ng}$ et al (8) und $H$ sia et al (5) keine oder nur unwesentlich erhöhte Aktivitäten der Galaktose-1-phosphat-Uridylyltransferase in den Erythrocyten finden konnten, berichteten Rosner et al (6) und Schuppisser et al (9) über Steigerungen der Aktivität dieses Enzymes in den roten Blutzellen.

Wegen dieser Diskrepanz haben wir nochmals die Aktivität der Galaktose-1-phosphat-Uridylyltransferase im Erythrocytenhämolysat von 30 Mongoloiden untersucht, zumal uns seit 1971 eine neue Bestimmungsmethode (10) zur Verfügung steht.

\section{Methodik}

\section{Prinzip}

Als Maß für die Aktivität des Enzyms Galaktose-1-phosphatUridylyltransferase wird die in der Zeiteinheit verbrauchte Menge UDPG verwendet. Nach Abstoppen der Meßreaktion bestimmt man die verbleibende UDPG-Menge mit Hilfe von UDPGDehydrogenase (E. C. 1.1.1.22) und NAD ${ }^{+}$im optisçhen Test:
1. Gal-1-P + UDPG $\rightleftharpoons$ UDP-Gal + Glu-1-P

2. UDPG $+2 \mathrm{NAD}^{+} \longrightarrow$ UDP-Glucuronsäure + $2 \mathrm{NADH}+2 \mathrm{H}^{+}$

Die methodischen Einzelheiten werden nach den von Haschemian (10) gegebenen Richtlinien durchgefüht.

Reagenzien

1. Glycin-Puffer, $1 \mathrm{~mol} / 1, \mathrm{pH} 8,7$

2. Glycin-Puffer, $0,2 \mathrm{~mol} / 1, \mathrm{pH} 8,9$

3. UDPG-Lösung, $10,5 \mathrm{mmol} / 1$

4. UDPG-Glycinmischung: 1 Teil UDPG-Lösung, 4 Teile GlycinPuffer, $1 \mathrm{~mol} / 1$

5. Gal-1-P-Lösung, $8 \mathrm{mmol} / 1$

6. NAD-Lösung, 13,6 mmol/1

7. UDPG-Dehydrogenase (Suspension in AmmoniumsulfatLösung, 3,2 mol/1, Aktivität $3 \mathrm{U} / \mathrm{ml}$ )

Die Lösungen 3. bis 6. werden mit Präparaten der Firma Boehringer, Mannheim, angesetzt, die Enzymsuspension bezogen wir ebenfalls von der Firma Boehringer.

\section{Hämolysat}

Zentrifugation von heparinisiertem Vollblut (20 E Heparin/ml) bei $2000 \mathrm{~g} 15 \mathrm{~min}$ lang in der Kälte, absaugen des überstehenden Plasmas und der Leukocytenschicht (weitgehende Vollständigkeit wird dadurch erreicht, daß auch noch die obere Erythrocytenschicht mit erfaßt wird). Zweimaliges Waschen der Erythrocyten in $9 \mathrm{~g} / 1 \mathrm{NaCl}-\mathrm{Lösung}(1 \mathrm{Vol}$. Erythrocytenkonzentrat in 2 Vol. NaCl-Lösung); Zentrifugation bei $2000 \mathrm{~g} 15 \mathrm{~min}$ lang;

1) Durchgefuhrt mit freundlicher Unterstützung des Ministerpräsidenten des Landes Nordrhein-Westfalen - Landesamt für Forschung. 
mischen des gewaschenen Erythrocytenkonzentrates mit dest. Wasser (1 Vol. Erythrocytenkonzentrat mit 2 Vol, dest. Wasser); zweimaliges Einfrieren dieses Hämolysates im Methanolbad bei $-28^{\circ} \mathrm{C}$ mit anschließendem Auftauen; zerstören von NAD durch 10 min Vorinkubation bei $37^{\circ} \mathrm{C}$ zur Inaktivierung der UDPGEpimerase (E.C. 5.1.3.2).

Die Hämoglobinbestimmung im Hämolysat erfolgte nach Kampen und Zijlstra (13).

\section{Meßreaktion}

Folgende Mengen werden im Eisbad in Zentrifugenröhrchen pipettiert:

\begin{tabular}{lcc} 
& Reaktionsansatz & Leerwert \\
\hline UDPG-Glycin & $0,2 \mathrm{ml}$ & $0,2 \mathrm{ml}$ \\
Gal-1-P & $0,1 \mathrm{ml}$ & - \\
dest. Wasser & - & $0,1 \mathrm{ml}$ \\
Hämolysat & $0,2 \mathrm{ml}$ & $0,2 \mathrm{ml}$
\end{tabular}

Anschließend inkubiert man $15 \mathrm{~min}$ im Wasserbad bei $37^{\circ} \mathrm{C}$. Nach Abstoppen der Reaktion durch Einstellen der Röhrchen ins Eisbad ( $5 \mathrm{~min}$ ) wird in jedes Röhrchen $1 \mathrm{ml} 9 \mathrm{~g} / 1$ eiskalte $\mathrm{NaCl}-\mathrm{Lösung}$ pipettiert.

Enteiweißen der Ansätze: Die mit Parafilm verschlossenen Röhrchen werden 2 min lang in ein siedendes Wasserbad gestellt und dabei intermittierend geschüttelt. Danach Zentrifugation bei $2000 \mathrm{~g} 15 \mathrm{~min}$ lang und anschließende Filtration des enteiweiß ten Überstandes.

\section{Indika torreaktion}

In Küvetten mit kritischem Volumen von weniger als $1 \mathrm{ml}$ $(d=1 \mathrm{~cm})$ werden folgende Substanzen pipettiert:

\begin{tabular}{lcc} 
& Reaktionsansatz & \multicolumn{1}{c}{ Leerwert } \\
\hline Glycin-Puffer, $0,2 \mathrm{~mol} / 1$ & $0,7 \mathrm{ml}$ & $0,7 \mathrm{ml}$ \\
NAD & $0,1 \mathrm{ml}$ & $0,1 \mathrm{ml}$ \\
Filtrat & $0,2 \mathrm{ml}$ & $0,2 \mathrm{ml}$
\end{tabular}

Nach Mischen Ablesen der Extinktion $\left(E_{1}\right)$ bei $340 \mathrm{~nm}$. Anschließend wird jeder Probe 0,02 ml UDPG-DehydrogenaseSuspension zugefügt und nach Stillstand der Reaktion (nach etwa $15 \mathrm{~min}$ ) $\mathrm{E}_{2}$ abgelesen.

\section{Berechnung}

$\frac{\Delta \mathrm{E} \cdot \mathrm{K}}{\mathrm{Hb}(\mathrm{g} / \mathrm{ml}) \cdot 60}=\mu \mathrm{mol}$ UDPG $\cdot$ Verbrauch $/ \mathrm{min} \cdot \mathrm{gHb}$

$\mathrm{K}=12,3 ; \mathrm{K}$ setzt sich zusammen aus: Testvolumen $(1,02)$, Verdünnungsfaktor $(7,5)$ des Hämolysates, Extinktionskoeffizient von NADH $\left(6,22 \mathrm{~cm}^{2} / \mu \mathrm{mol}\right.$ bei $\left.340 \mathrm{~nm}\right)$, Schichtdicke $(1 \mathrm{~cm})$ Probevolumen $(0,2 \mathrm{ml})$, Inkubationszeit (1/4 Stunde), sowie Faktor 2, da pro Mol UDPG 2 mol NADH gebildet werden: Eine Einheit des Enzyms ist dann die Menge, die den Umsatz von $1 \mu \mathrm{mol}$ UDPG/min verursacht.

\section{Patienten}

Es werden 20 männliche Patienten mit Down-Syndrom (im Alter zwischen 2 und 31 Jahren) und 10 weibliche (im Alter zwischen 8 und 37 Jahren), sowie 30 alters- und geschlechtsentsprechende Gesunde untersucht. Alle Patienten mit M. Down sind in Heimen fur geistig Behinderte oder Sonderschulen als Mongoloide erfaßt.

\section{Ergebnisse}

Von den untersuchten 30 Paaren wurden aus folgenden Gründen bei der statistischen Auswertung zwei Paare ausgesondert:
1. Bei einer 16-jährigen, weiblichen Patientin mit M. Down ergab die Galaktose-1 phosphat-Uridylyltransferasebestimmung eine Aktivität von $333 \mathrm{mU} / \mathrm{g}$ $\mathrm{Hb}$, ein Wert, welcher innerhalb des Normbereiches für Gesunde lag. Da es sich in diesem Fall möglicherweise um einen Translokationsmongolismus, bei dem bisher keine erhöhten Enzymaktivitäten gefunden wurden (1) handelte, wurde der hier gefundene Wert nicht berücksichtigt.

2. Eine Galaktose-1-phosphat-Uridylyltransferaseaktivität von $1116 \mathrm{mU} / \mathrm{g} \mathrm{Hb}$ von einem männlichen Mongoloiden erwies sich bei der statistischen Beurteilung nach Nalimov signifikant als Ausreißer: $\mathrm{r}^{*}=2,99$ (mehr als $\mathrm{r}_{99}$ ).

Das so bereinigte Material ergab für 28 (19 männliche und 9 weibliche) Mongoloide eine Galaktose-1-phosphatUridylyltransferaseaktivität von durchschnittlich $690 \pm$ 103,33 mU/g Hb (Extremwerte: 500-883 mU்/g Hb), und für 28 Gesunde einen arithmetischen Mittelwert von $291 \pm 103,50 \mathrm{mU} / \mathrm{g} \mathrm{Hb}$ (Extremwerte: $133-483 \mathrm{mU} / \mathrm{g}$ $\mathrm{Hb})$. An Hand der gefundenen Ergebnisse zeigte sich in den Erythrocyten der Patienten mit M. Down eine Aktivitätssteigerung des untersuchten Enzymes um das durchschnittlich 2,7-fache gegenüber den Vergleichswerten bei einer Standardabweichung von $\pm 1,05$.

Zur Beurteilung der Mittelwertdifferenzen zwischen den zusammengehörenden Datenpaaren von Patienten mit Down-Syndrom und den Gesunden errechneten wir mit Hilfe des Differenzen-t-Testes einen hochsignifikanten

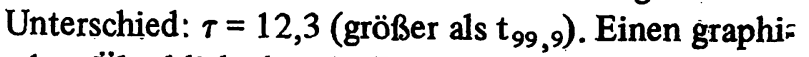
schen Überblick über die Ergebnisse zeigt die Abbildung 1.

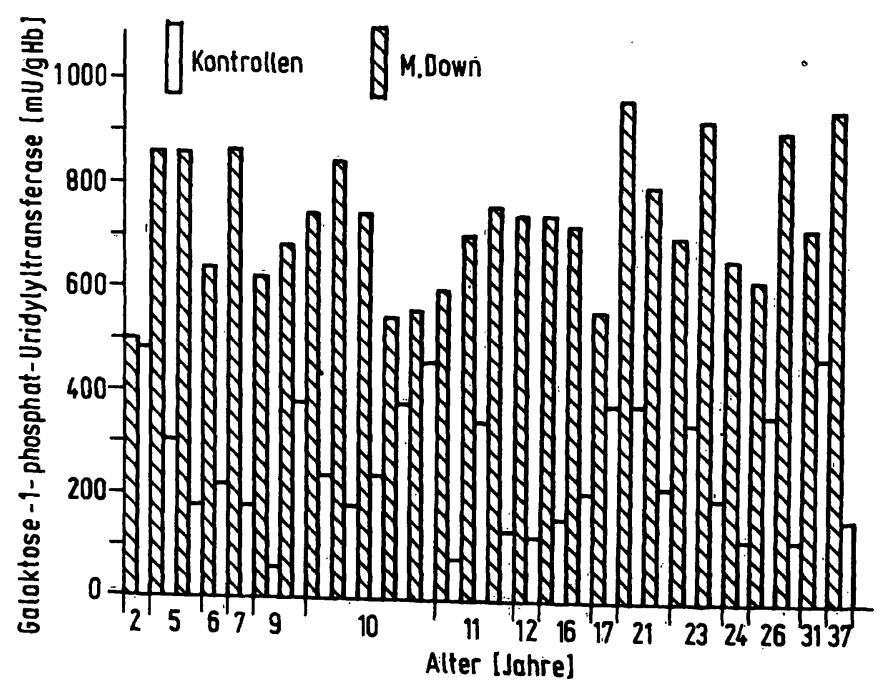

Abb. 1. Galaktose-1-phosphat-Urydyltransferaseaktivität bei 28 Patienten mit Down-Syndrom im Vergleich zu alters- und geschlechtsentsprechenden Kontrollen, auf der Abszisse von links nach rechts mit steigendem Alter angeordnet. 


\section{Diskussion}

Der von Hsia et al (5) vorgebrachte und nachgewiesene Einwand, daß die Aktivitätssteigerung der Galaktose-1phosphat-Uridylyltransferase im Vollblut ausschließlich auf intrazelluläre Leukocytenenzymaktivitätssteigerung zurückzuführen ist und die Erythrocyten nicht betrifft, konnte aus methodischen Gründen durch unsere Befunde nicht bestätigt werden, da wir zur Untersuchung nur Erythrocytenhämolysat einsetzten.

Bei der Interpretation unserer Ergebnisse wurde der Differenzen-t-Test zur Beurteilung der Mittelwerte der beiden zusammengehörenden Datenpaare benutzt: Er ergab mit $\tau=12,3$ (mehr als $t_{99,9}$ ) einen hochsignifikanten Unterschied der Meßwerte. Im Durchschnitt betrug die Enzymaktivität bei Patienten mit Down-Syndrom das 2,7-fache der Aktivität der Kontrollgruppe. Dieser Befund spricht gegen die bisher mehrfach diskutierte „Ein Gen/Ein Enzym"-Hypothese $(6,9)$, da hiernach nur ein Verhältnis von 1,5:1 erwartet werden darf. Erklärt werden könnte dagegen der von uns gefundene 1,7-fache Enzymaktivitätsanstieg gegenüber der Norm mit dem bei Mongoloiden offensichtlich vorhandenen niedrigeren mittleren Alter der peripheren Blutzellen $(11,12)$.

Nicht unerwähnt bleiben soll die Tatsache, daß der von uns gefundene Mittelwert bei Gesunden von $291 \mathrm{mU} / \mathrm{g}$ $\mathrm{Hb}$ um $93 \mathrm{mU} / \mathrm{g} \mathrm{Hb}$ niedriger lag als bei der Originalbeschreibung (10).

Dies könnte verursacht sein durch heterozygote Merkmalsträger für Galaktosämie in unserer Vergleichsgruppe.

\section{Literatur}

1. Hsia, D. Y. Y., Justice, P., Smith, G. F. \& Dowben, R. M. (1971), Amer. J. Dis. Child. 121, 153-161.

2. Lenz, W. (1967), in Humangenetik, (Beckex, P. E. Hrsg.) 345-346, G. Thieme-Verlag, Stuttgart, 8. Auflage.

3. Brandt, N. J. (1962), Lancet II, 837

4. Brandt, N. J., Fr $\phi l$ land, A., Mikkelsen, M., Nielsen, A. \& Tolstrup, N. (1963), Lancet II, 700-703

5. Hsia, D. Y. Y., Inouye, T., Wong, P. \& South, A. (1964), N. Engl. J. Med. 270, 1085-1088.

6. Rosner, F., Ong, B. H., Paine, R. S. \& Mahanand, D. (1965), N. Engl. J. Med. 273, 1356-1361

7. Meliman, W. J., Oski, F. A. Tedesco, T. A., Maciera-Coelho, A. \& Harris, H. (1964), Lancet II, 674-675

8. Ng, W. G., Bergren, W. R. \& Donnell, G. N. (1964), Clin. Chim. Acta 10, 337-343.

9. Schuppisser, R., Joss, E. \& Richterich, R. (1967), Schweiz. Med. Wochenschr. 97, 1540-1542.

10. Haschemian, G. (1971), Inaug.-Dissertation, Münster.

11. Hook, E. B. \& Engel, R. R. (1964), Lancet I, 112.

12. Naiman, J. L., Oski, F. A. \& Mellman, W. J. (1965), Lancet I, 821

13. Kampen, E. J. van, \& Zijlstra, W. G. (1961) Clin. Chim. Acta 6, 538-544.

Prof. Dr. F. Menne Physiol. Chem. Inst. 44 Münster Waldeyerstr. 15 